\title{
Interleukin-6 (174G/C) Gene Polymorphism and Serum Levels of IL-6, their Association with Risk of Obesity in Iraqi Childhood Populations
}

\author{
Manal M. Kadhim ${ }^{1}$, Intesar A. Salman ${ }^{2}$ \\ ${ }^{1}$ Professor of Clinical Immunology, Department of Medical Microbiology, College of Medicine, University of \\ Al-Qadisiyah, Diwaniya, Iraq, ${ }^{2}$ Assistant Lecturer, Department of Medical Microbiology, College of Medicine, \\ University of Al-Qadisiyah, Babil, Iraq
}

\begin{abstract}
Background: Childhood obesity is one of the most serious public health challenges of the 21 st century.

Aim: The present study was conducted to investigate the association of $C D 14$ genotype and serum levels of IL-6 with risk of ischemic stroke.

Method : A total of 60 patients with obesity were included in the study who were admitted to hospital from the period between March to August 2020, and other groups consist of 60 apparently healthy individuals. A five $\mathrm{ml}$ of blood samples were collected, $3 \mathrm{ml}$ of each sample for polymerase chain reaction amplification and detection of IL- 6 technique. The remaining $(2 \mathrm{ml})$ for IL-6 serum levels by ELISA assay procedure.
\end{abstract}

Results: $I L-6(-174 \mathrm{G} / \mathrm{C})$ genotype was significantly associated with increased risk of obesity $(\mathrm{P}=0.004)$. However, IL-6 serum levels were higher in subjects with GG genotype compared with those with CG or CC genotype $(\mathrm{P}<0.001)$.

Conclusion: A significant correlation between IL-6 genotype and IL-6 levels were higher level in subjects with GG genotype compared with those with CG or CC genotype.

Keyword: Childhood Obesity, BMI, IL-6.

\section{Introduction}

Childhood obesity is one of the most serious public health challenges of the 21 st century(1). It is widely known that obesity reduces child health-related quality of life and is associated with several health and social consequences. These include the risk of premature death, as well as developing diabetes, cancer, heart disease ${ }^{(2)}$. It also causes undesirable psychological consequences, such as anxiety, depression, sleep disorders and low self-esteem, which affects the social and educational relationships of children ${ }^{(3)}$. A pooled analysis of 2416 population-based measurement studies showed that from 1975 to 2016 there was a rising trend in children's and adolescent's body mass index (BMI). In 2016, 124 million children and adolescents, aged 5-19 years, were estimated to suffer from obesity worldwide and 213 million were overweight ${ }^{(4)}$. The number of obese children and adolescents (aged five to 19 years) worldwide has risen tenfold in the past four decades. If current trends continue, more children and adolescents will be obese than moderately or severely underweight by $2022^{(5)}$. Researchers believe that the increased prevalence of obesity is the result of changes in the life- style of societies, such as the inactivity, unhealthy eating patterns, collapse of energy balance, short sleep duration, increased use of fast food and animal proteins, and increased use of technology ${ }^{(6)}$. Interleukin- 6 (IL-6) is an immune-modulator pro-inflammatory cytokine, secreted by many types of cells, mainly $\mathrm{T}$ cells, macrophages, endothelial cells, smooth muscle cells, adipocytes, and hepatocytes (7). IL-6 is critical in the inflammatory signaling pathway and is involved in the development of obesity and insulin resistance ${ }^{(\mathbf{8})}$. 
Furthermore, IL-6 regulates/stimulates production of cell adhesion molecules, acute phase protein, and mediates the release of other cytokines that amplify the inflammatory response which influence the function of adipose tissue ${ }^{(9)}$. The human $I L-6$ gene is located on chromosome $7 \mathrm{p} 21$, and the $-174 \mathrm{G} / \mathrm{C}$ polymorphism consists of a single nucleotide change from $\mathrm{G}$ to $\mathrm{C}$ at position -174 in the promoter region ${ }^{\mathbf{( 1 0 )}}$. The association of the $I L-6-174 G / C$ polymorphism with obesity risk has been evaluated in several genetic studies. Some studies have suggested that $I L-6-174 G / C$ increased the obesity risk ${ }^{(11)}$, while some found no association between IL6-174G/C and obesity ${ }^{(\mathbf{1 2})}$.

\section{Materials and Method}

The current study was carried on 60 patients $(25$ males, 35 females) age range between 4-18 years from March to August 2020. Other groups consist of 60 apparently healthy individuals ( 30 male and 30 female) without any history of systemic disease were clinically considered as healthy also included in this study as a control group. We excluded patients have obesity secondary to a genetic or medical condition, such as diabetics, polycystic ovarian syndrome, hypothyroidism, Cushing's syndrome, growth hormone deficiency, insulinoma, hypothalamic disorders (e.g., Froelich syndrome, Bardet-Biedl syndrome, Prader-Willi syndrome), or medication use (e.g., antipsychotics). A five $\mathrm{ml}$ of blood samples were collected by vein puncture using disposable syringes under aseptic technique $3 \mathrm{ml}$ of each sample were transferred into with EDTA tube and immediately frozen at $-20 \mathrm{C}$ till further use to avoid repeated thawing and freezing for polymerase chain reaction amplification and detection of $I L-6$ (RFLPPCR)technique. The remaining $(2 \mathrm{ml})$ were transferred intosterile test tubes (Plain tube) and allow sample to clot for few minutes at room temperature then followed by separation of serum from the clot by centrifugation for 10 minutes at 2500 r.p.m. and stored at $-20^{\circ} \mathrm{C}$ for ELISA assay procedure. This study was in agreement with ethics of Al-Imam Al Sadiq Teaching Hospital and verbal informed consent was obtained from all participants.

\section{Results}

Sixty Childhood obesity patients included in this study. The socioeconomic and lifestyle variables distribution of the study population are summarized in Table (1). There were no significant differences between daily duration of sleep and risk for obesity $(\mathrm{P}=0.138)$.
The present study found a statistically significant between family income and obesity $(p=0.007)$. The frequency distribution of obesity further increased with the number of hours per day spent watching television (33.4\% of childhood patients spent more than four hours per day in watching television), although the relation was not significant $(\mathrm{P}=0.175)$. The frequency distribution of childhood patients and healthy controls according to physical activity indicated the prevalence of obesity was lower among childhood who practiced physical activity $(\mathrm{P}=0.003)$. Distribution of $I L-6-174 G>C$ polymorphism was detected by RFLP-PCR technique, at this locus there are three genotypes, CG,GG and CC with band sizes 198/140/158 bp, 140/158 bp and 198 bp respectively, table (2), figure (1). The genotype distribution had no deviation from Hardy-Weinberg equilibrium in all study groups and agree with the reports of Ibrahim et $\mathrm{al}^{\mathbf{( 1 3 )}}$. Both groups were comparable in Median serum concentration IL-6 table (3), figure (2). Median serum concentration of IL-6 in obesity patients was significantly higher than that of healthy control 127.32 (62.43) ng/L versus 89.44 (29.75) ng/L and P-value was $(\mathrm{P}<0.001)$.Median serum concentration of IL-6 were statistically significantly higher in patients bearing the GG genotype $(\mathrm{p}<0.001)$ compared with heterozygotes (CG) and those homozygous for the CC genotype, and this indicate serum level of IL-6 was highly significant associated to $I L-6$ genotype polymorphism in childhood patients group, table (4).

\section{Table 1: Frequency Distribution of Patients with Obesity and Healthy Control according to some Socioeconomic and lifestyle variables.}

\begin{tabular}{|c|c|c|c|}
\hline Characteristic & Patients $n=60$ & Control $n=60$ & $\mathbf{P}$ \\
\hline \multicolumn{4}{|c|}{ Sleep duration (h/day) } \\
\hline$<8, \mathrm{n}(\%)$ & $19(31.7 \%)$ & $26(43.3 \%)$ & \multirow{3}{*}{0.138} \\
\hline $9-10, n(\%)$ & $17(28.3 \%)$ & $20(33.3 \%)$ & \\
\hline$>10, n(\%)$ & $24(40 \%)$ & $14(23.4 \%)$ & \\
\hline \multicolumn{4}{|c|}{ Average family income } \\
\hline Low, $n(\%)$ & $9(15 \%)$ & $24(40 \%)$ & \multirow{3}{*}{0.007} \\
\hline Medium, $n(\%)$ & $21(35 \%)$ & $18(30 \%)$ & \\
\hline High, n (\%) & $30(50 \%)$ & $18(30 \%)$ & \\
\hline \multicolumn{4}{|c|}{ Elevision viewing (h/day) } \\
\hline$<1, \mathrm{n}(\%)$ & $14(23.3 \%)$ & $24(40 \%)$ & \multirow{4}{*}{0.175} \\
\hline $1-2, n(\%)$ & $8(13.3 \%)$ & $9(15 \%)$ & \\
\hline $2-4, \mathrm{n}(\%)$ & $18(30 \%)$ & $15(25 \%)$ & \\
\hline$>4, \mathrm{n}(\%)$ & $20(33.4 \%)$ & $12(20 \%)$ & \\
\hline \multicolumn{4}{|l|}{ Physical activity } \\
\hline Yes & $26(43.3 \%)$ & $42(70 \%)$ & \multirow{2}{*}{0.003} \\
\hline No & $34(56.7 \%)$ & $18(30 \%)$ & \\
\hline
\end{tabular}


Table (2): Distribution of $I L-6-174 G / C$ Genotype and Alleles Frequency

\begin{tabular}{|c|c|c|c|c|c|c|c|}
\hline Genotype & Controls $(n=60)$ & Patients $(n=60)$ & OR & 95\% Confidence interval & p & PF & EF \\
\hline \multicolumn{8}{|c|}{$I L-6-174 \mathrm{G} / \mathrm{C}$} \\
\hline GG & 15 & 26 & 4.189 & $1.66-10.57$ & 0.002 & $\ldots \ldots$ & 0.52 \\
\hline $\mathrm{CG}$ & 16 & 22 & 3.23 & $1.31-8.43$ & 0.01 & $\ldots \ldots$ & 0.45 \\
\hline $\mathrm{CC}$ & 29 & 12 & Reference & Reference & Reference & $\ldots \ldots$ & Reference \\
\hline \multicolumn{2}{|c|}{ Overall P value } & \multicolumn{6}{|c|}{0.004} \\
\hline \multicolumn{8}{|c|}{ Alleles Frequency } \\
\hline G & 46 & 74 & 2.59 & $1.54-4.35$ & \multirow{2}{*}{0.001} & & 0.378 \\
\hline $\mathrm{C}$ & 74 & 46 & 0.386 & $0.23-0.65$ & & 0.378 & $\ldots \ldots$ \\
\hline
\end{tabular}

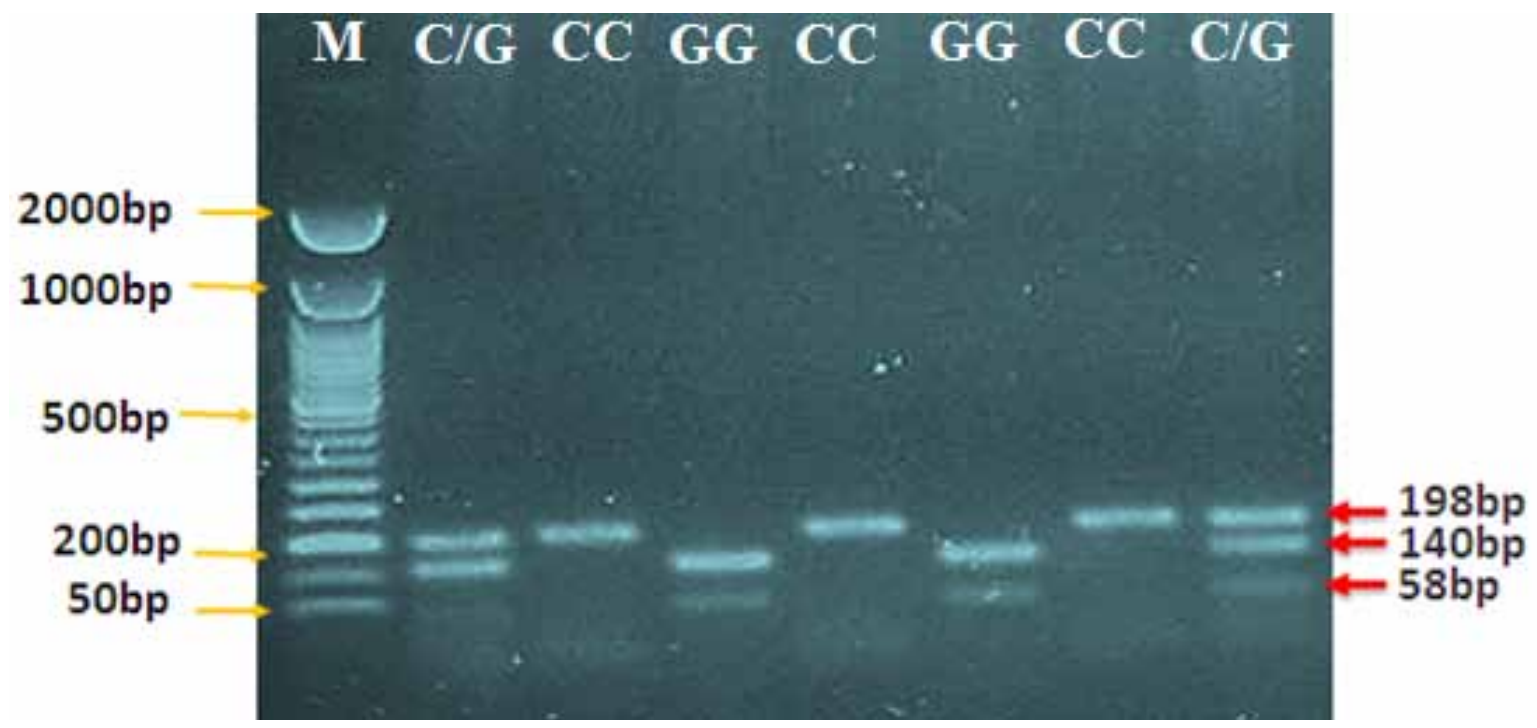

Figure (1): Agarose gel electrophoresis image that show the RFLP-PCR product analysis of IL-6 gene-174G/C (rs1800795) gene polymorphism by using SfaNI restriction enzyme in $2.5 \%$ agarose gel. Where M: marker (2000-50bp). Lane (CC) wild type homozygote, the product undigested by restriction enzyme and still at 198bp bands. Lane (GG) mutant type homozygote that show digested by restriction enzyme into 140bp and 58bp bands. Lane (C/G) heterozygote, the product digested by restriction enzyme into $198 \mathrm{bp}, 140 \mathrm{bp}$ and $58 \mathrm{bp}$ bands.

Table (3): The compassion between the study groups regarding IL-6 level

\begin{tabular}{|l|c|c|c|}
\hline IL-6(ng/L) & Patients $\mathbf{n}=\mathbf{6 0}$ & Control $\mathbf{n}=\mathbf{6 0}$ & P \\
\hline Range & $77.3-230$ & $42.72-145.33$ & 0.001 \\
Median (IQR) & $127.32(62.43)$ & $89.44(29.75)$ & (S) \\
\hline
\end{tabular}

Table (4): The Median Serum Interleukin-6 in patients with obesity and control subjects according to Interleukin-6 genotype

\begin{tabular}{|l|c|c|}
\hline IL-6 POLY & Patients groups n $=60$ & Control groups n $=60$ \\
\hline GG & $149.86(60.7)$ & $113(35.66)$ \\
\hline CG & $122.87(37.57)$ & $90.33(13.79)$ \\
\hline CC & $93.4(10.41)$ & $77.62(45.57)$ \\
\hline P & $<0.001$ & $<0.001$ \\
& HS $\dagger$ & HS \\
\hline
\end{tabular}




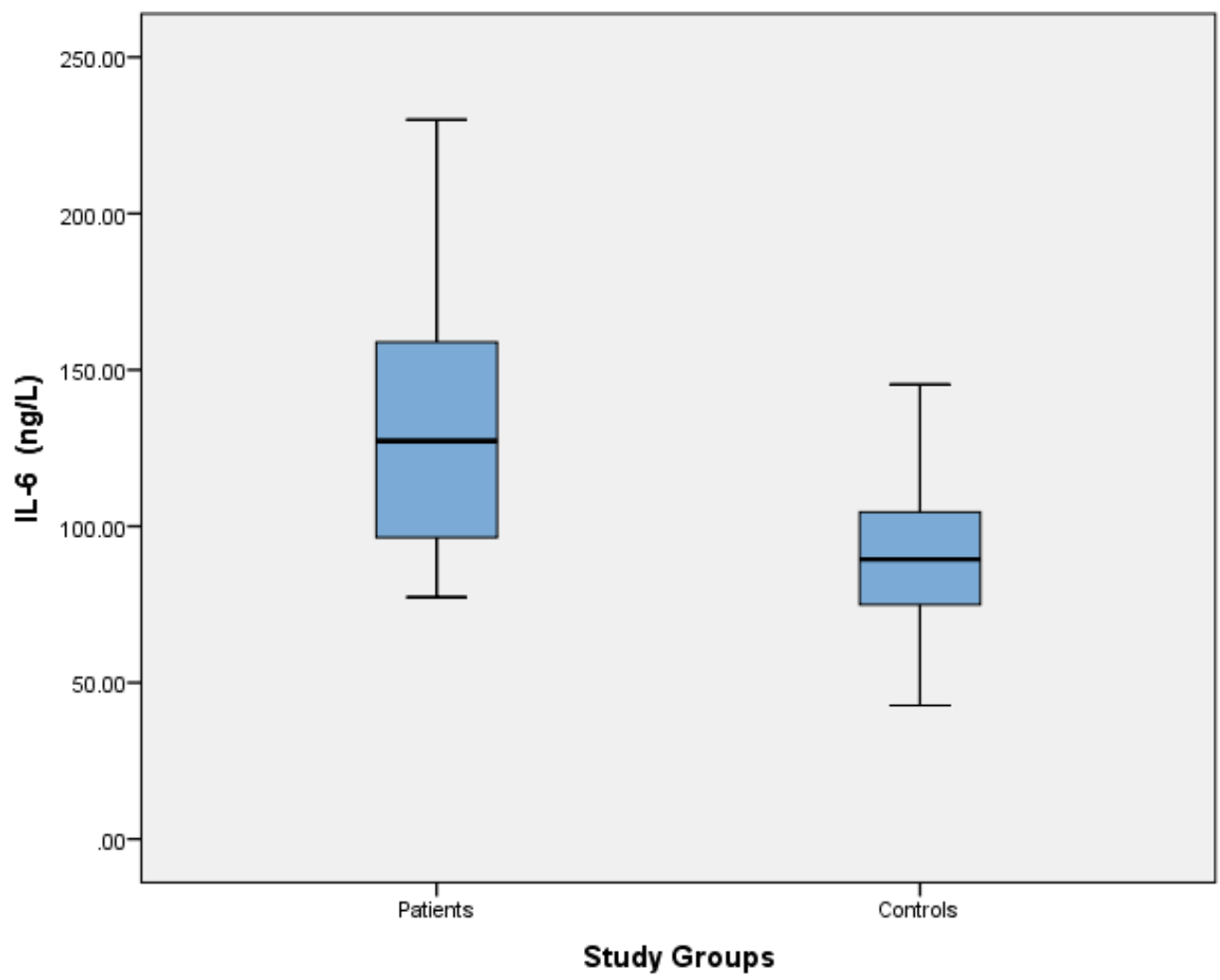

Figure (2): Distribution of patients with obesity and control subjects according to the level of Serum Interleukin-6

\section{Discussion}

Table (1) revealed that $24(40 \%)$ of the patients with obesity with sleep duration more than ten hours and $14(23.4 \%)$ of healthy control with sleep duration more than ten hours, and this result suggested increased obesity with increased sleep duration, but these results indicated no significant difference in the frequency distribution of patients and control subjects according to sleep duration $(\mathrm{P}=0.138)$. The results of present study agree with results of El-Kabbaoui et al., ${ }^{(14)}$. (2018), which indicated 78 (38.6\%) of patients has had more than ten hours sleep duration and these result indicate no significant association between the daily duration of sleep and risk for overweight or obesity $(\mathrm{P}=0.75)$.We found a statistically significant relation between family income and risk of obesity, where the obesity increasing with increasing family income $30(50 \%)$ of patients have had high average family income. Several explanations have been proposed. The low prevalence of obesity in groups of low socioeconomic status in developing countries is related to food scarcity, patterns of high energy expenditure and the greater capacity of the elite to obtain adequate food supplies ${ }^{(\mathbf{1 5})}$. The correlations may be due to the benefits of economic growth, notably better access to food and high energy expenditure by poorer social groups, difficulty in acquiring more expensive, less energy-dense foods and a trend towards less leisure time and fewer opportunities for exercise ${ }^{(\mathbf{1 4})}$. A similar finding was reported in a study of Moroccan adults, in which family income, used as a determinant of socioeconomic status, was strongly associated with obesity ${ }^{(16)}$.

Table (1) revealed that $20(33.4 \%)$ of the patients with obesity was had more than four hours Television viewing, although watching television daily for $\geq 4 \mathrm{~h}$ was not significantly associated with obesity in our study when compared to healthy controls $(p=0.175)$. Watching television may reduce energy expenditure by replacing physical activity and also increase snacking, which is further encouraged by advertisements for energy-dense foods ${ }^{(17)}$. Our results agree with results of El-Kabbaoui et al., ${ }^{(14)}$, which indicated non-significant correlation between watching television daily for $\geq 4$ $\mathrm{h}$ with the risk of childhood for obesity $(\mathrm{p}=0.32)$. The present results found inverse statistically significant relation between the prevalence of obesity and physical 
activity $(\mathrm{P}=0.003)$, where the frequency distribution of childhood obesity patients according to physical activity was as following : $26(43.3 \%)$ patients having physical activity and 34 (56.7 \%) don't having physical activity. Inadequate physical activity has been hypothesized to be an important contributing factor to the development of childhood obesity. The influence on energy expenditure can explain this relationship. Physiologically, sedentary posture lowers energy expenditure, and this in turn promotes weight gain ${ }^{(\mathbf{1 8 )}}$.A review of the influence of physical activity on adiposity among 5-18-yearolds showed that adiposity was reduced and aerobic capacity increased with more time spent in intense physical activity ${ }^{(\mathbf{1 9})}$. The present results in consistence with results of several studies, For instance, a study in Saudi Arabia showed that intense physical activity was inversely associated with adolescent obesity ${ }^{(20)}$, and a strong negative association was reported between vigorous physical activity and total and central body fat in Spanish adolescents ${ }^{(21)}$.

Obesity is caused by several genetic, metabolic, social, and environmental factors. Among these internal and external factors, the contribution of genetic factors has been recognized widely, but the genes involved have not been fully elucidated ${ }^{(22)}$. Genetic variants, especially functional polymorphisms in the promoter region of genes, may alter the function and expression of genes associated with energy intake and energy expenditure. Several indications of the linkage between single nucleotide polymorphisms (SNPs) and obesity phenotypes have been found ${ }^{(23)}$. Interleukin-6 polymorphisms have been investigated in many populations for associations with various chronic diseases. For example, single-nucleotide polymorphisms (SNPs) rs1800797(-597 G/A), rs1800796 (-572G/C) and rs1800795 $(-174 \mathrm{G} / \mathrm{C})$, located in the promoter region of IL6, have been shown to be associated with obesity and metabolic traits in different ethnic groups (24). Accordingly, $I L-6-174 G>C$ were selected and their genetic associations with obesity risk were evaluated using RFLP PCR analysis to identify if genetic variants in IL-6 might be a potentially genetic marker to predict the susceptibility of obesity. Overall, highly significant differences can be found in the distribution of the genotype and allele frequencies of IL-6 (rs1800795) between the obesity patients and control subjects $(\mathrm{P}=$ 0.004). The present results consistence with results of Nelson et al., ${ }^{(25)}$, which indicated IL-6-174G $>$ C polymorphism was highly associated with the risk of obesity.
The results of present study showed high frequency of (GG) genotype among patients when compared to healthy controls, these result identify (GG) genotype is associated with increased risk of obesity with statistically significant $(P=0.002)$, and may be considered as the etiological factor for obesity. The results of present study consistence with result of Yang et $\mathrm{al}^{\mathbf{( 2 6 )}}$, which found the GG genotype was significantly higher in patients with obesity than in those without $(\chi 2=10.63, p=0.005)$. The present study are relatively conflicting with previous reports which revealed that the $I L-6$ rs 1800795 SNP in the genotype GG play a protective role and present predominated in the control group (95.3\%) with a statistically significant difference compared to that of obese children $(28.2 \%)^{(\mathbf{1 3})}$. Moreover, the present result revealed frequency of allele $\mathrm{C}$ was more in healthy control compared to obesity patients. Also showed that (CC) genotype frequency in patients (20\%) lower than in healthy controls $(48.3 \%)$ and this results disagree with results Oana et al ${ }^{(27)}$, which found that the G allele at $I L-6$ as being more common in lean subjects and observed the $\mathrm{C}$ allele to be associated with indices of obesity.

Higher serum concentration of IL-6 may be due to its important role in pathophysiology of obesity. The adipose tissue is a major source of circulating IL-6, and the excessive secretion of IL- 6 appears to be directly related to obesity ${ }^{(28)}$. IL-6 also can influence adipocyte function, lipid metabolism and thus play a major role in the development of obesity ${ }^{(24)}$. Other theory Saied IL-6 exerts strong effects on hormonal balance and may induce some endocrnological disturbances. It is suggested that IL-6 may affect the increase of free fatty acids level. IL- 6 concentration is elevated in patients with lipid disorders and insulin resistance ${ }^{(29)}$. The increased IL-6 levels in obese individuals may result in a state of insulin resistance and increased risk of cardiovascular complication ${ }^{(30)}$. Our results agree with results of Ibrahim et al., ${ }^{(13)}$, which found The mean serum level of IL-6 in obese children was significantly higher as compared to those of control children $(7.7 \pm 0.46 \mathrm{pg} / \mathrm{mL}$ and $5.46 \pm 0.40 \mathrm{pg} / \mathrm{mL}$ respectively $(\mathrm{P}=0.003)$.

Table (4), showed significant association between serum IL-6 levels and IL-6 Polymorphism in patients with obesity $(p<0.001)$, were we found higher median serum IL-6 levels in the GG genotype 149.86 (60.7) $\mathrm{ng} / \mathrm{L}$ as compared with $\mathrm{CG}$ or CC genotype (122.87 (37.57)ng/L and 93.4 (10.41) ng/L respectively). The present study found higher promoter activity of the rs1800795 G-allele compared with the C-allele, where 
CC homozygotes genotype had lower serum IL-6 levels when compared with G-allele carriers in both obesity patients and healthy controls. This suggests that rs1800795 G-allele carriers with higher levels of IL6 have a higher risk for obesity.

\section{Conclusions}

Our results confirm clinically relevant relationship of $I L-6$ gene polymorphism with risk of obesity. Childhood Patients who undergone obesity showed a higher level of IL-6 than healthy control. In this study we found a significant correlation between $I L-6$ genotype and IL-6 levels were higher level in subjects with GG genotype compared with those with $\mathrm{CG}$ or $\mathrm{CC}$ genotype.

Financial Disclosure: There is no financial disclosure.

Conflict of Interest: None to declare.

Ethical Clearance: All experimental protocols were approved under the Department of Medical Microbiologyand all experiments were carried out in accordance with approved guidelines.

\section{References}

1. Mohamed S. Childhood Obesity: Epidemiology, Determinants, and Prevention. Journal of Nutritional Disorders \& Therapy. 2015; 5: 156.

2. Gies I, Al-Saleem B, Olang B. Early childhood obesity: a survey of knowledge and practices of physicians from the Middle East and North Africa. BMC Pediatr. 2017; 17(1):115.

3. Hudda $M$, Nightingale $C$, Donin A. patterns of childhood body mass index (BMI), overweight and obesity in south Asian and black participants in the English national child measurement programmer: effect of applying BMI adjustments standardizing for ethnic differences in BMI-body fatness associations. Int J Obes. 2018; 42(4):662.

4. Barca-Gómez L, Abdeen Z, Hamid Z. NCD Risk Factor Collaboration (NCD-RisC). Worldwide trends in body-mass index, underweight, overweight, and obesity from 1975 to 2016 : a pooled analysis of 2416 population-based measurement studies in 128.9 million children, adolescents, and adults. Lancet. 2017; 390 (10113): 2627-42.

5. WHO. Tenfold increase in childhood and adolescent obesity in four decades: new study by Imperial College London and WHO. Saudi Med J. 2017; 38
(11): 1162-1163.

6. Rahmani A, Sayehmiri K, Asadollahi K. Investigation of the prevalence of obesity in Iran: a systematic review and meta-analysis study. Acta Med Iran. 2015; 53(10):596-607.

7. Rodrigues K, Pietrani N, Bosco A. IL-6, TNF- $\alpha$, and IL-10 levels/polymorphisms and their association with type diabetes mellitus and obesity in Brazilian individuals. Arch Endocrinol Metab. 2017; 61(5):438-446.

8. Underwood P.C., Chamarthi B., Williams J.S., et al., (2012): Replication and meta-analysis of the geneenvironment interaction between body mass index and the interleukin- 6 promoter polymorphism with higher insulin resistance. Metabolism. 61:667-71.

9. Fisman E, Tenenbaum A. The ubiquitous interleukin-6: a time for reappraisal. Cardiovasc Diabetol. 2010; 9:62.

10. Hu M, Yu Z, Luo D. Association between $-174 \mathrm{G}>\mathrm{C}$ polymorphism in the IL-6 promoter region and the risk of obesity A meta-analysis. Medicine. 2018; 97:33.

11. De Filippo G, Rendina D, Moccia F. Interleukin-6, soluble interleukin-6receptor/interleukin-6 complex and insulin resistance in obese children and adolescents. J Endocrinol Invest 2015; 38:33943.

12. Bienertova-Vasku J, Bienert P, Tomandl J. No association of defined variability in leptin, leptin receptor, adiponectin, proopiomelanocortin and ghrelin gene with food preferences in the Czech population. Nutr Neurosci.2008; 11:2-8.

13. Ibrahim O, Gabre A, Sallam S. Influence of Interleukin-6 (174G/C) Gene Polymorphism on Obesity in Egyptian Children. Open Access Macedonian Journal of Medical Sciences. 2017; 5(7):831-835.

14. El Kabbaoui M, Chda A, Bousfiha A. Prevalence of and risk factors for overweight and obesity among adolescents in Morocco. East Mediterr Health J. 2018; 24(6):512-521.

15. Monteiro CA, Moura EC, Conde WL Socioeconomic status and obesity in adult populations of developing countries: a review. Bull World Health Organ. 2004; 82(12):940-6.

16. El Rhazi K, Nejjari C, Zidouh A. Prevalence of obesity and associated sociodemographic and lifestyle factors in Morocco. Public Health Nutr. 
2011; 14(1):160-7.

17. Lowry R., Wechsler H., Galuska DA. Television viewing and its associations with overweight, sedentary life-style, and insufficient consumption of fruits and vegetables among US high school students: differences by race, ethnicity, and gender. J Sch Health. 2008; 72(10):413-21.

18. Hamilton MT, Hamilton DG, Zderic TW. Role of Low Energy Expenditure and Sitting in Obesity, Metabolic Syndrome, Type 2 Diabetes, and Cardiovascular Disease. Diabetes. 2007; 56(11):2655-67.

19. Parikh T, Stratton G. Influence of intensity of physical activity on adiposity and cardiorespiratory fitness in 5-18 year olds. Sports Med. 2011; 41(6):477-88.

20. Al-Hazzaa HM,Abahussain NA, Al-Sobayel HI. Lifestyle factors associated with overweight and obesity among Saudi adolescents. BMC Public Health. 2012; 16;12:354.

21. Moliner-Urdiales D, Ruiz J, Ortega F. HELENA Study Group. Association of objectively assessed physical activity with total and central body fat in
Spanish adolescents; the HELENA Study. Int J Obes. 2009; 33(10):1126-35.

22. Pigeyre M, Saqlain M, Turcotte M. Obesity genetics: insights from the Pakistani population. Obes Rev.2018; 19:364-80.

23. Valladares M, Obregon A, Chaput J. Association between genetic variants of the clock gene and obesity and sleep duration. J PhysiolBiochem.2015; 71:855-60.

24. Boeta-Lopez K, Duran J, Elizondo D. Association of interleukin-6 polymorphisms with obesity or metabolic traits in young Mexican-Americans. Obesity Science \& Practice.2017; 138; 85-96.

25. Nelson J, Handa P, Aouizerat B. Increased parenchymal damage and steatohepatitis in Caucasian non-alcoholic fatty liver disease patients with common IL1B and IL6 polymorphisms. Aliment PharmacolTher. 2016; 44:1253-64.

26. Yang X, Feng L, Li C Li Y. Association of IL6-174G/C and $-572 \mathrm{C} / \mathrm{G}$ polymorphisms with risk of young ischemic stroke patients. Gene 2014; 539:256-262. 